\title{
Toward understanding the role of the neuron-specific BAF chromatin remodeling complex in memory formation
}

\author{
Kwang-Yeon Choi, Miran Yoo and Jin-Hee Han
}

The long-term storage of memory requires the finely tuned coordination of intracellular signaling with the transcriptional, translational and epigenetic regulations of gene expression. Among the epigenetic mechanisms, however, we know relatively little about the involvement of chromatin remodeling-dependent control of gene expression in cognitive brain functions, compared with our knowledge of other such mechanisms (for example, histone modifications and DNA methylation). A few recent studies have implicated the Brm/Brg-associated factor (BAF) chromatin-remodeling complex, a mammalian homolog of the yeast Swi/Snf complex, in neuronal structural/functional plasticity and memory formation. The BAF complex was previously known to have a critical role in neurodevelopment, but these recent findings indicate that it also contributes to both cognitive functions in the adult brain and human mental disorders characterized by intellectual disability. In this review, we provide a brief overview of the BAF complexes, introduce recent research findings that link their functions to memory formation, and speculate on the yet-unknown molecular mechanisms that may be relevant to these processes.

Experimental \& Molecular Medicine (2015) 47, e155; doi:10.1038/emm.2014.129; published online 3 April 2015

\section{INTRODUCTION}

How can our brain store memories for a long time? Neuroscientists have made massive efforts to answer this question in recent decades, but the molecular mechanisms underlying long-term memory remain elusive. It has been well established that long-term memory requires de novo gene expression through transcription and translation. The newly synthesized proteins are thought to support the synaptic functional/ structural plasticity needed to encode and store memory. Importantly, persistent epigenetic changes in gene expression patterns have been proposed as a key molecular mechanism underlying the formation of long-lasting memories.

In eukaryotes, the long DNA strand is highly compacted into chromatin structures, which must be modified to allow active transcription. The chromatin consists of DNA that is wrapped around histone octamers called nucleosomes, and then further condensed into higher-order structures. Covalent modifications of histone tails (for example, acetylation and methylation) can alter the compaction state of chromatin. The highly compacted state (heterochromain) hampers the ability of transcription factors and the transcriptional machinery to access the promoters of target genes, whereas the relaxed (euchromatin) state allows active transcription. In response to neuronal activity during learning experiences, the gene expression patterns of neurons may be regulated by several different epigenetic mechanisms, including DNA methylation, histone acetylation and ATP-dependent chromatin remodeling. The regulations of gene expression by DNA methylation ${ }^{1,2}$ and histone acetylation $^{3,4}$ are well known to be essential for long-term memory formation. However, the comparable role of chromatin remodeling-dependent regulation has only recently begun to be explored. ${ }^{5}$

There are four main classes of chromatin remodeling complexes: Swi/Snf, ISWI, INO80 and Mi2/CHD. ${ }^{6}$ The $\mathrm{Brm} / \mathrm{Brg}$-associated factor complex (BAF complex), which is a mammalian homolog of the yeast Swi/Snf complex, can evict or slide nucleosomes to regulate the accessibility of DNA in an ATP-dependent manner. It can also exchange histone variants and induce conformational changes in the chromatin structure. The BAF complex is a multisubunit complex consisting of at least 15 subunits, ${ }^{7}$ including an ATPase (either Brg or Brm). BAF170, BAF155 and BAF47 are the functional core subunits; they all, including Brg, are sufficient to induce remodeling via nucleosome shift in vitro. ${ }^{8}$ Based on the combination of

Department of Biological Sciences, KAIST Institute for the BioCentury (KIB), Korea Advanced Institute of Science and Technology (KAIST), Daejeon, Korea Correspondence: Professor J-H Han, Department of Biological Sciences, KAIST Institute for the BioCentury (KIB), Korea Advanced Institute of Science and Technology (KAIST), 291 Daehak-ro, Yuseong-gu, Daejeon 305-701, Korea. 
additional BAF subunits, a BAF complex may be classified as an embryonic stem cell BAF, a neuronal progenitor BAF, a neuronal BAF (nBAF) or a polybromo-associated BAF complex. Diverse combinations of BAF complexes enable the cell-type- or developmental-stage-specific regulation of gene expression from a single copy of the genome. ${ }^{9}$ It was recently revealed that the nBAF complex and its specific subunit, BAF53b, have critical roles in long-term memory. ${ }^{5}$ In this review, we focus on the functions of this complex. Comprehensive reviews of the other BAF complexes are available in the literature. $^{9-12}$

\section{NEURON-SPECIFIC BAF COMPLEXES}

\section{Identification of neuron-specific BAF53b}

In 2002, Olave et al. ${ }^{13}$ first identified BAF53b (also known as hArpN $\alpha$, Actl6b); it is a homolog of BAF53a (hArpN $\beta$, Actl6a), which was cloned in 1998. ${ }^{14}$ BAF53b and BAF53a are classified as actin-related protein 4 . BAF53b comprises 426 amino acids (aa) and shares $87 \%$ identity (93\% similarity) with BAF53a, ${ }^{15}$ but is highly divergent through $\mathrm{N}$-terminal aa40-82 (called subdomain 2). ${ }^{13}$ BAF53a and BAF53b are mutually exclusive components of the BAF complex. BAF53b is specifically found in nBAF complexes, and its expression is restricted to postmitotic neurons. ${ }^{13}$

\section{Neurodevelopmental role of BAF complexes}

It is well known that BAF complexes have essential functions in development. The combinatorial assembly of the various subunits produces diverse BAF complexes that have specific functions during development. ${ }^{9}$ For example, the characteristic embryonic stem cell BAF complexes are BAF155 homodimers, lack BAF170 and have Brg (not Brm) as their ATPase; ${ }^{16}$ these complexes are necessary and sufficient for the self-renewal and pluripotency of embryonic stem cells. ${ }^{16,17}$ The neuronal progenitor BAF complex, which is characterized by the inclusion of BAF170 and the presence of either Brg or Brm, is necessary for the transition from the embryonic stem cell stage to the neuroprogenitor stage. ${ }^{18}$ When neural progenitor cells differentiate into post-mitotic neurons, they switch from expressing BAF53a to expressing BAF53b, and trade out other nBAF-specific subunits; for example, BAF45a/d and SS18 are exchanged for $\mathrm{BAF} 45 \mathrm{~b} / \mathrm{c}$ and $\mathrm{Ca}^{2+}{ }_{\text {-responsive transcriptional }}$ coactivator (CREST; also known as SS18L1), respectively.,15,18 This switching is mediated by the expression of the microRNAs (miRNAs), miR9* and miR124. ${ }^{19}$ These miRNAs are known targets of RE1-silencing transcription factor (REST), which acts with a corepressor to repress neuronal genes in non-neuronal cells. ${ }^{19}$ Repression of REST allows the expression of these miRNAs. The BAF53a messenger RNA transcript has binding sites for miR9* and miR124 in its $3^{\prime}$ untranslated region; miRNA binding translationally inhibits the expression of BAF53a which represses BAF53b expression. Thus, the miRNA-mediated blockade of BAF53a expression subsequently activates the expression of BAF53b. Conversely, mutations designed to inhibit the binding of these miRNAs were shown to prolong BAF53a expression and attenuate BAF53b expression in post-mitotic neurons. ${ }^{19}$ The importance of these miRNAs in neurodevelopment was highlighted by the finding that miR9* and miR124 are sufficient to direct the differentiation of human fibroblasts into neurons. ${ }^{20,21}$

\section{BAF53b has a critical role in activity-dependent dendritic outgrowth}

Given its post-mitotic-neuron-specific expression pattern, BAF53b was thought to have an important functional role in the adult brain. To test this, BAF53b knockout mice were generated. ${ }^{15}$ Homozygous Baf53b knockout mice were found to be viable; however, only $\sim 12 \%$ of pups survived to adulthood, and the mice showed behavioral abnormalities (for example, hyperactivity). ${ }^{15}$ The latter characteristics make it difficult to perform behavioral tests on the knockout mice. Cultured Baf53b $\mathrm{b}^{-1-}$ neurons showed deficits in activitydependent dendritic outgrowth. Similar results were seen following the short-hairpin RNA-mediated knockdown of other nBAF complex subunits (for example, Brg, BAF45b and BAF57). ${ }^{15}$ These findings suggest that the deficit in activity-dependent outgrowth was due to nBAF complex dysfunction. Furthermore, the results from experiments using a chimeric BAF53b protein containing subdomain 2 of BAF53a (and vice versa) suggested that the highly divergent subdomain 2 of BAF53b is necessary and sufficient for activity-dependent dendritic remodeling. ${ }^{15}$ The function of BAF53b in dendritic remodeling is conserved in invertebrates. In Drosophila, the knockdown of Bap55, a homolog of BAF53a/b, impaired the proper targeting of olfactory dendrites. ${ }^{22}$ Interestingly, the Bap55 mutant phenotype could be rescued by human BAF53a or BAF53b in contrast to failure of rescue by BAF53a in rodent. ${ }^{15,22}$

\section{THE ROLE OF BAF53B IN LEARNING AND MEMORY}

\section{Synaptic plasticity}

The efforts to elucidate the function of BAF53b in the adult brain made significant progress when Vogel-Ciernia et al. ${ }^{5}$ generated CaMKII promoter-driven Baf53b dominant negative transgenic mice, thereby avoiding the developmental impacts of BAF53b knockout. Based on previous studies using BAF53a,, 23 overexpression of mutant BAF53b with deletion of the C-terminal hydrophobic domain (aa 323-333; BAF53b $\Delta H D$ ) was assumed to competitively inhibit the interaction between endogenous wild-type BAF53b and other subunits of the nBAF complex. ${ }^{5,23}$ Thus, the deletion mutant was thought to act as a dominant negative form of BAF53b. Both BAF53b $\Delta \mathrm{HD}$ mice and $\mathrm{Baf} 53 \mathrm{~b}^{+/-}$mice showed deficits in the long-term forms of hippocampus-dependent memory (for example, object location and contextual fear memories), whereas auditory fear memory, which is amygdala-dependent, was not affected in either type of transgenic mouse. ${ }^{5}$ This study also measured long-term potentiation (LTP) in hippocampal slices using theta burst stimulation (TBS) in both types of transgenic mice. Baf $53 b^{+/-}$mice showed impairment in the maintenance of LTP, while $B a f 53 b \Delta \mathrm{HD}$ transgenic mice yielded different results depending on the level of BAF53b $\Delta \mathrm{HD}$ expression: Baf $53 b \Delta \mathrm{HD}^{\text {low }}$ mice 
(having a relatively lower level of expression) showed LTP impairments following subthreshold 5 TBS but not following suprathreshold 10 TBS, whereas Baf $53 b \Delta \mathrm{HD}^{\text {high }}$ mice (having a relatively higher level of expression) showed LTP impairment and an abnormally enhanced early-LTP response following 10 TBS. Furthermore, a paired-pulse facilitation experiment demonstrated that Baf53b $\Delta \mathrm{HD}^{\text {high }}$ mice had enhanced excitability in the presynaptic portion of their LTP. This phenomenon suggests that overexpression of $B a f 53 b \Delta \mathrm{HD}$ may trigger a gain-of-function.

\section{Structural plasticity}

If the function of BAF53b is important for synaptic plasticity and long-term memory formation, we might next ask: What is the underlying molecular mechanism? Based on the observation that BAF53b is essential for activity-dependent dendritic outgrowth, ${ }^{15}$ researchers examined neuronal structural changes in the CA1 neurons of Baf53b $\Delta \mathrm{HD}^{\text {high }}$ mice, and found that the ratio of thin-type to mushroom type-spines was decreased at baseline, but the total spine density was unchanged. ${ }^{5}$ The observed increase in mushroom-type spines was consistent with a previous report of a similar increase following Brm knockdown. ${ }^{24}$ It is possible that this abnormality in dendritic spine structure at baseline caused the observed deficits in longterm memory. However, it is not yet clear whether BAF53b participates in learning-induced structural plasticity.

There is evidence that the induction of LTP in a single spine with glutamate uncaging induces rapid enlargement of the spine volume via $\mathrm{N}$-methyl-D-aspartate (NMDA) receptor activation. ${ }^{25-27}$ This local regulation of spine volume crucially involves remodeling of the actin cytoskeleton, which is regulated by local Rho GTPases (for example, RhoA and Cdc42). ${ }^{27}$ There is also evidence that tetanic stimulation-induced LTP shifts the F-actin/G-actin equilibrium toward F-actin in dendritic spines. ${ }^{28}$ The experimental blockade of actin polymerization was found to inhibit both spine volume changes ${ }^{26,28}$ and LTP. ${ }^{29,30}$ TBS was shown to induce phosphorylation of PAK and its effector, Cofilin, specifically in activated synapses within a few minutes post stimulation. ${ }^{31,32}$ Indeed, the portion of pCofilin-positive spines is increased on learning. ${ }^{33}$ VogelCiernia et al. ${ }^{5}$ observed mice at $7 \mathrm{~min}$ after TBS, and found that the increase in pCofilin-positive spines was impaired in $B a f 53 b^{+/-}$mice compared with wild-type mice. This suggests that activity-dependent actin cytoskeleton signaling is disrupted in $B a f 53 b^{+/-}$mice. Indeed, RNA-sequencing (RNA-seq) revealed that several genes known to mediate the actin cytoskeleton are dysregulated in Baf53b $3 b^{+/-}$mice. $^{5}$

miRNAs as possible regulators of actin filaments and spine volume

Among the genes found to be dysregulated in $B a f 53 b^{+/-}$mice, miR132 and miR134 are notable because they are known to be involved in the Cofilin-dependent regulation of the actin cytoskeleton. The expression level of miR132 was increased after learning in wild-type mice but not in $\mathrm{Baf}_{5} 3 \mathrm{~b}^{+/-}$mice, ${ }^{5}$ and accumulating evidence has implicated miR132 in structural changes among neurons. For example, miR132 was found to be necessary and sufficient to induce neurite outgrowth in cultured neurons. ${ }^{34}$ Mild (but not robust) elevation of miR132 was found to enhance memory in rodents, ${ }^{35,36}$ and miR132 was shown to be transiently induced by pCREB, BDNF and learning. ${ }^{34,37}$ Furthermore, miR132 was observed to inhibit the translation of p250GAP, ${ }^{38}$ derepressing Racl and activating the Pak/LIMK1 pathway to phosphorylate Cofilin (Figure 1). ${ }^{32,39,40}$ Thereafter, pCofilin ${ }^{36}$ (the inactive form of the protein) can stabilize the actin filament by inhibiting its depolymerization. Although miR132 is known to be transcriptionally regulated by $\mathrm{pCREB},{ }^{34}$ the activitydependent phosphorylation of CREB (which is upstream of miR132) was not altered in Baf53 $3 b^{-1-}$ mice compared with wild type. ${ }^{15}$ Thus, it is likely that the altered transcription of miR132 in $\mathrm{Baf} 53 \mathrm{~b}^{+/-}$mice results from the abnormalities in chromatin remodeling caused by reduced BAF53b function.

Unlike miR132, miR134 was found to be slightly, but significantly, downregulated on learning in wild-type mice but not Baf $53 b^{+/-}$mice $(P=0.015$, Figure 1$) .{ }^{5}$ The miR134 is enriched in synaptodendritic compartments. ${ }^{41}$ In the resting state, miR134 restrains the local protein synthesis of LIMK1, maintaining a low expression level of this protein. When BDNF is upregulated, however, local protein synthesis of LIMK1 occurs via the mTOR pathway. ${ }^{41}$ LIMK1 phosphorylates Cofilin in the local synapse, and pCofilin-positive spines promote polymerization of the actin cytoskeleton and enlargement of the spine. These mechanisms allow the actin cytoskeleton to be remodeled in a temporally and spatially accurate manner. Because each miRNA typically impacts hundreds of target messenger RNAs, the nBAF complexmediated regulation of miRNA transcription could enable the further refinement of neuronal gene expression. Therefore, miR132 and miR134 are plausible candidate effectors for the function of BAF53b in the activity-dependent regulation of spine morphology. Together, these findings on BAF53b suggests that the chromatin remodeling mediated by the BAF complex in neurons of the adult brain may have crucial roles in synaptic plasticity and long-term memory storage, perhaps by regulating the activity-dependent changes in spine morphology via actin signaling.

\section{COUNTERPART PROTEINS THAT INTERACT WITH BAF53B}

BAF53b and beta-actin bind to the helicase-SANT-associated domains of $\mathrm{Brg}$ or $\mathrm{Brm},{ }^{42}$ but the binding proteins that interact with BAF53b outside the nBAF complex have not yet been fully elucidated. In yeast two-hybrid screens using aa 40-82 of BAF53b (which are highly divergent with respect to BAF53a) as bait, Oma et al. ${ }^{43}$ found that the transcriptional co-repressor, $\mathrm{C}$-terminal binding protein (CtBP), interacts with BAF53b. The authors further showed that this interaction between BAF53b and CtBP contributes to regulating gene expression via the known interaction between Brg and glucocorticoid receptor, in that CtBP suppressed glucocorticoid response elementassociated transcription in the presence of BAF53b, but not 


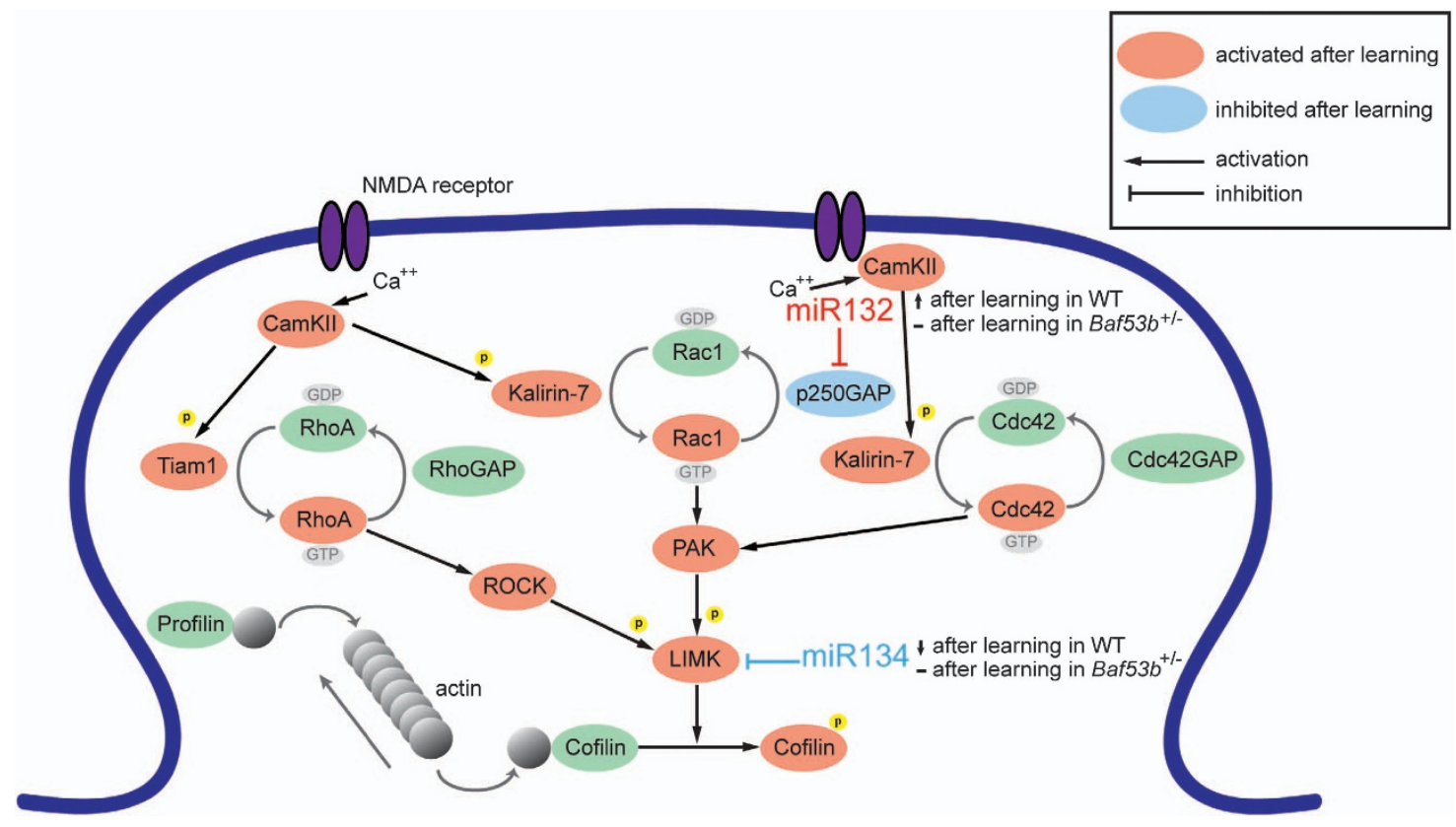

Figure 1 The microRNAs (miRNAs) that show post-learning dysregulation in Baf53 $b^{+/-}$mice regulate actin remodeling via Cofilin. This schematic depicts the proposed model for how regulators of the actin cytoskeleton in dendritic spines are influenced by the loss of BAF53b. The influx of calcium through the N-methyl-D-aspartate (NMDA) receptor directly and indirectly activates small GTPase exchange factors, activating RhoA, Rac1 and Cdc42 and leading to the accumulation of pCofilin in neuronal spines. Following this stimulation, miR132 and miR134 can regulate the level of pCofilin. The red circles indicate post-learning upregulation in wild-type mice, while the blue circles indicate post-learning downregulation in $B a f 53 b^{+/-}$mice. Yellow circled p, phosphorylation.

aa 40-82-deleted BAF53b. ${ }^{43}$ Notably, an immunoprecipitation study failed to detect any interaction between BAF53b and CtBP, ${ }^{15}$ suggesting that the interaction between BAF53b and CtBP may be relatively weak and/or transient, appearing only on stimulation.

\section{SIGNALING UPSTREAM OF THE NBAF COMPLEX Calcium signaling pathways}

We might next ask: which upstream signaling pathways activate the BAF complex in neurons? The evidence suggests that calcium signaling may be involved. Aizawa et al. ${ }^{44}$ identified the CREST, when screening for calcium-induced transactivators. Interestingly, CREST knockout mice are a phenocopy of Baf53 $b^{-1-}$ mice: Crest $^{-1-}$ mice are viable, but only $20 \%$ survive to adulthood and their cultured neurons show deficits in calcium-dependent dendritic growth. ${ }^{44}$ CREST itself does not have any DNA binding ability, suggesting that other DNAbinding proteins likely act as binding partners for CREST to induce gene expression. Indeed, CREST has been shown to interact with the nBAF complex by binding to Brg. ${ }^{45}$ Although BAF53b does not directly interact with CREST, it was found to be necessary for the recruitment of CREST onto target gene promoters. $^{15}$

The interactions of CREST, Brg and CBP regulate $c$-fos expression, providing clues toward understanding how the nBAF complex regulates target genes. ${ }^{45}$ In the resting state, the nBAF complex docks to the Sp1 binding site of the $c$-fos promoter and $\mathrm{Brg}$ recruits $\mathrm{HDAC1}$ via $\mathrm{Rb}$, thereby repressing the transcription of $c$-fos. In the active state, in contrast, calcium stimulation induces the calcineurin-mediated dephosphorylation of $\mathrm{Rb}$ and subsequent HDAC1 release, allowing acetylation of the nucleosome. Concurrently, CaMKIV phosphorylates the coactivator, CBP, which directly interacts with CREST, leading to the transcriptional activation of $c$-fos.

Calcium signaling is transmitted into the nucleus via NFAT, CaMK, MAPK and PKA signaling, and free calcium transient from synapse can directly arrive at nucleus. ${ }^{46}$ Synaptic transmission increases the local calcium concentration in postsynaptic spines through NMDA and voltage-gated calcium channels, and a large amount of calcium is reserved in the endoplasmic reticulum (ER). Glutamate activates mGluR, which is coupled to phospholipase C (PLC), causing PLC to hydrolyze phosphatidylinositol 4,5-bisphosphate $\left(\mathrm{PIP}_{2}\right)$ into diacylglycerol and inositol 1,4,5-triphosphate $\left(\mathrm{IP}_{3}\right)$. Upon binding of $\mathrm{IP}_{3}, \mathrm{IP}_{3}$-sensitive calcium channels in the ER release calcium from the ER lumen into the cytoplasm. Because the nucleus communicates with the ER, an elevation of calcium at local synapses may produce a calcium wave that eventually arrives at the nucleus via the ER. ${ }^{47}$

\section{Inositol phosphate signaling}

Inositol phosphate signaling is another potential upstream signaling pathway that might regulate nBAF complex activity. There is not yet any in vivo evidence supporting this notion, but several in vitro studies have shown that inositol phosphates functionally regulate the BAF complexes. ${ }^{14,48}$ In lymphocytes, $\mathrm{PIP}_{2}$ is sufficient to target the BAF complex to chromatin, ${ }^{14}$ and other related inositol phosphates have been suggested to 


\section{a \\ Wu et al. 2007}
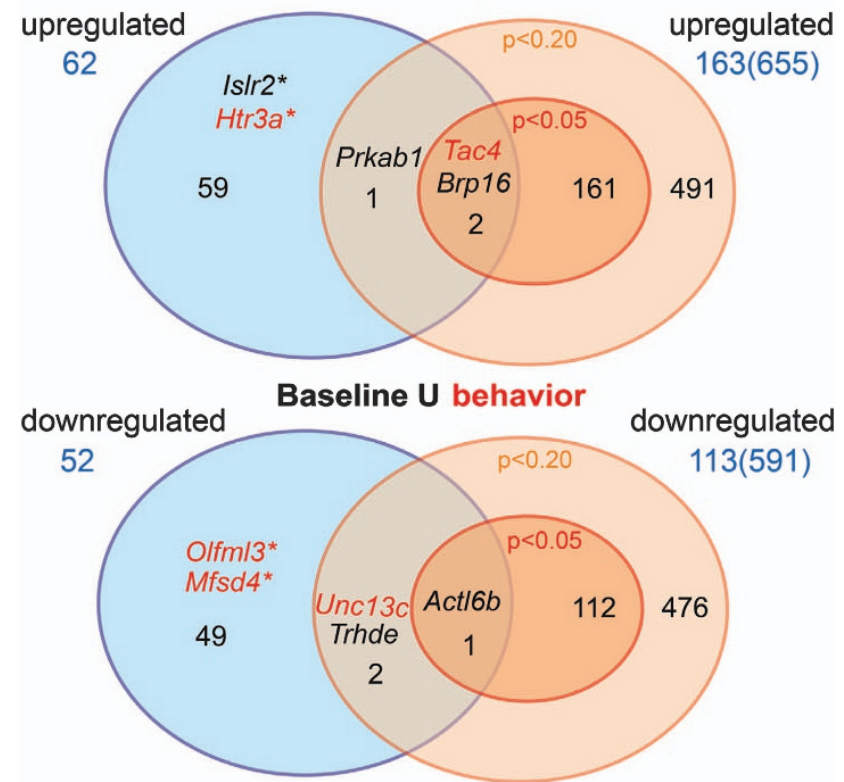

b

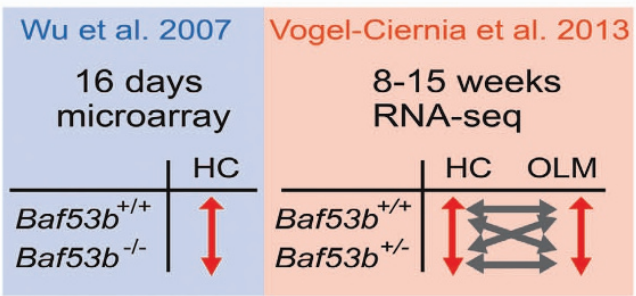

Figure 2 Overlap of target genes identified by the two highthroughput experiments. (a) Venn diagram showing concordant and discordant BAF53b target genes identified in two recent highthroughput studies. Of the 62 genes found to be upregulated in the microarray analysis, only 2 were also shown to be differentially expressed in the RNA-sequencing (RNA-seq) experiment. Among the downregulated genes identified by the microarray analysis, only Actl6b (BAF53b) was shared between the two data sets. When the $P$-value cutoff was loosened, only one and two genes were additionally shared among the up and downregulated genes, respectively. Colors and symbols: U, math symbol for union; red font, dysregulated after learning; black font, dysregulated at baseline; *, oppositely dysregulated in the two studies $(P<0.20$ and $P \geqslant 0.05$ ). (b) Comparison of the two studies. The highthroughput platforms differed, as did the ages and genotypes of the utilized mice. Vogel-Ciernia et al. ${ }^{5}$ made six comparisons, whereas Wu et al. ${ }^{15}$ made only two. Here, we looked at genes that were dysregulated at baseline or after learning. HC, home cage; OLM, object location memory.

modulate the activity of chromatin remodeling complexes in vivo. ${ }^{48,49}$ In yeast, $\mathrm{IP}_{4}$ and $\mathrm{IP}_{5}$ stimulate the SWI/SNF complex, whereas $\mathrm{IP}_{6}$ inhibits nucleosome remodeling. ${ }^{48,49}$ Rando et al. ${ }^{50}$ used the results from various experiments to propose a model for the $\mathrm{PIP}_{2}$-mediated activation of the BAF complex. First, they used $\mathrm{PIP}_{2}$ micelles and light scattering to show that $\mathrm{PIP}_{2}$ binds the BAF complex. Then, they examined the binding of actin, which is known to bind at two distinct $\mathrm{C}$-terminal regions of $\mathrm{Brg}$. $\mathrm{PIP}_{2}$ sensitively decreased the binding affinity of one site while the other remained bound. The authors therefore concluded that $\mathrm{PIP}_{2}$ may allow the uncapped BAF complex to bind the pointed ends of actin filaments or the filament branch points through the newly exposed actin surface and BAF53a/b.

\section{HIGH-THROUGHPUT STUDIES FOR TARGET GENES OF BAF53B}

Regarding the target genes of the nBAF complex, two highthroughput studies have been conducted in rodent brains. $\mathrm{Wu}$ et al. ${ }^{15}$ performed microarray experiments to identify 114 genes that were dysregulated in Baf53b homozygous knockout mice using tissue samples were taken from the hippocampi of 16-day-old Baf53 $3^{-/}$(P16) and control mice. Vogel-Ciernia et $a .^{5}$ used RNA-seq to investigate dysregulated genes in hippocampal tissues of adult $B a f 53 b^{+/-}$and wild-type littermate control mice, examining both baseline levels and learning-induced changes in the gene expression profiles. Although the two studies differed in their experimental settings (see Figure 2b), we speculated that the genes reported in common between these two studies could be potential target genes for BAF complex-mediated regulation. Toward this end, we compared the results from these microarray and RNA-seq studies.

Of the 62 genes found to be upregulated in the microarray data, only 2 (Tac4 and Brp16) were also found in the lists of dysregulated genes identified by RNA-seq at baseline or after learning in Baf53b $b^{+/-}$mice (Figure 2a). Among the downregulated genes, only one gene (Actl6b) was found in both data sets. Even when we expanded the $P$-value cutoff from 0.05 to 0.20 in the RNA-seq data, only one (Prkab1) and two additional genes (Unc13c and Trhde) were found to be shared in the up and downregulated data sets, respectively. Some genes (for example, Islr2, Htr3a, Olfml3 and Mfsd4) were even found to be dysregulated in opposite directions $(0.05 \leqslant P<0.20)$ (Figure 2a). In particular, Ngef (also known as Ephexin1), which is thought to be a key target gene of both BAF53b and $\mathrm{CREST}^{15}$ in regulating dendritic remodeling on stimulation, ${ }^{15}$ was unaltered in the RNA-seq results from $\mathrm{Baf}_{5} 3 \mathrm{~b}^{+/-}$mice at baseline and post learning, ${ }^{5}$ whereas it was significantly downregulated according to the microarray data.

A consistent finding in both studies was that immediate early genes, such as c-fos, zif268 (Egr1), Arc and Npas4, were not affected by the loss of BAF53b., ${ }^{5,15}$ Moreover, both studies found that genes involved in regulating the actin cytoskeleton (albeit disparate sets of such genes) were misregulated following the loss of BAF53b. For instance, Wu et al. ${ }^{15}$ identified Ngef (Ephexin1), Gsn (gelsolin, an actin-binding protein that is a key regulator of actin filament assembly and disassembly) and Racgap1 as being differentially expressed, whereas VogelCiernia et al. ${ }^{5}$ reported the dysregulations of miR132 (which inhibits p250GAP, as described in detail above), Citron (a Rho interacting kinase), Fhl2 (a member of the four-and-a-half LIM-only protein family, which has been implicated in linking signaling pathways to transcriptional regulation) and the upstream receptors, Grin2a, Grin2b and EphA4. 
How should we interpret the seeming discrepancies in the results of these two high-throughput experiments? One explanation is that the results may have been confounded by differences in the ages of the tested mice. In addition, brain tissues contain extremely heterogeneous cell populations (that is, >100 subtypes), making it far more difficult to study neuronal gene expression in vivo than it is to study other tissues or to examine cultured neurons in vitro. The heterogeneous cell types in the brain and the sparse distribution of the cells that are activated during learning make it very difficult to precisely determine cell-type-specific or learning-induced changes in gene expression profiles in the brain. In the future, gene expression profiling of defined neurons in specific brain regions should be used to better identify the target genes regulated by the nBAF complex at baseline and post learning.

\section{CONCLUDING REMARKS AND FUTURE PERSPECTIVES}

Epigenetic mechanisms for controlling gene expression have been critically implicated in long-lasting memory formation and various brain diseases characterized by intellectual disability. Traditionally, most of the relevant studies have focused on histone modifications and DNA methylation, with far less attention paid to the possible role of chromatin remodeling in memory formation. More recently, however, this has begun to change. Studies in transgenic mice with genetic deletions or dominant negative mutants of BAF53b (a subunit of the neuron-specific BAF chromatin-remodeling complex, nBAF) have revealed that the function of $\mathrm{nBAF}$ is crucial for synaptic plasticity and long-term memory formation in the hippocampus. It is particularly important to investigate the role of BAF53b-containing nBAF complexes in cognitive brain functions such as learning and memory, because such complexes are: (1) only found in post-mitotic neurons; (2) activated by calcium signals; (3) responsible for regulating many essential genes related to activity-dependent changes in spine morphology; and (4) associated with cognitive deficits when mutated. ${ }^{10,51}$ Although a great deal of research will be required to clarify how the nBAF complex regulates memory formation and how its mutation causes intellectual disability, some logical next steps would be to undertake acute genetic manipulation (either overexpression or knockdown) of BAF53b in the specific areas of adult brain that are relevant to memory formation, search for critical downstream effector molecules of the BAF complex and determine the detailed molecular mechanisms through which BAF complex-dependent chromatin remodeling mediates memory formation. These studies will provide important insights into the previously underexplored epigenetic regulation of enduring memory formation and human intellectual and cognitive disorders.

\section{CONFLICT OF INTEREST}

The authors declare no conflict of interest.

\section{ACKNOWLEDGEMENTS}

This study was supported by a grant from the Korean Health Technology R\&D Project, the Ministry of Health \& Welfare, Korea (A111293).

1 Miller CA, Sweatt JD. Covalent modification of DNA regulates memory formation. Neuron 2007; 53: 857-869.

2 Maddox SA, Watts CS, Schafe GE. DNA methyltransferase activity is required for memory-related neural plasticity in the lateral amygdala. Neurobiol Learn Mem 2014; 107: 93-100.

3 Guan J-S, Haggarty SJ, Giacometti E, Dannenberg J-H, Joseph N, Gao J et al. HDAC2 negatively regulates memory formation and synaptic plasticity. Nature 2013; 459: 55-60.

4 McQuown SC, Barrett RM, Matheos DP, Post RJ, Rogge GA, Alenghat T et al. HDAC3 is a critical negative regulator of long-term memory formation. J Neurosci 2011; 31: 764-774.

5 Vogel-Ciernia A, Matheos DP, Barrett RM, Kramár EA, Azzawi S, Chen Y et al. The neuron-specific chromatin regulatory subunit BAF53b is necessary for synaptic plasticity and memory. Nat Neurosci 2013; 16: 552-561.

6 Olave IA, Reck-Peterson SL, Crabtree GR. Nuclear actin and actin-related proteins in chromatin remodeling. Annu Rev Biochem 2002; 71: 755-781.

7 Staahl BT, Tang J, Wu W, Sun A, Gitler AD, Yoo AS et al. Kinetic analysis of npBAF to nBAF switching reveals exchange of SS18 with CREST and integration with neural developmental pathways. J Neurosci 2013; 33 10348-10361.

8 Phelan ML, Sif S, Narlikar GJ, Kingston RE. Reconstitution of a core chromatin remodeling complex from SWI/SNF subunits. Mol Cell 1999; 3: 247-253.

9 Ho L, Crabtree GR. Chromatin remodelling during development. Nature 2010; 463: 474-484.

10 Vogel-Ciernia A, Wood MA. Neuron-specific chromatin remodeling: a missing link in epigenetic mechanisms underlying synaptic plasticity, memory, and intellectual disability disorders. Neuropharmacology 2014; 80: $18-27$

$11 \mathrm{Wu} \mathrm{JI}$, Lessard J, Crabtree GR. Understanding the words of chromatin regulation. Cell 2009; 136: 200-206.

12 Yoo AS, Crabtree GR. ATP-dependent chromatin remodeling in neural development. Curr Opin Neurobiol 2009; 19: 120-126.

13 Olave I, Wang W, Xue Y, Kuo A, Crabtree GR. Identification of a polymorphic, neuron-specific chromatin remodeling complex. Genes Dev 2002; 16: 2509-2517.

14 Zhao K, Wang W, Rando OJ, Xue Y, Swiderek K, Kuo A et al. Rapid and phosphoinositol-dependent binding of the SWI/SNF-like BAF complex to chromatin after T lymphocyte receptor signaling. Cell 1998; 95: 625-636.

15 Wu JI, Lessard J, Olave IA, Qiu Z, Ghosh A, Graef IA et al. Regulation of dendritic development by neuron-specific chromatin remodeling complexes. Neuron 2007; 56: 94-108.

16 Ho L, Jothi R, Ronan JL, Cui K, Zhao K, Crabtree GR. An embryonic stem cell chromatin remodeling complex, esBAF, is an essential component of the core pluripotency transcriptional network. Proc Natl Acad Sci USA 2009; 106: 5187-5191.

17 Ho L, Ronan JL, Wu J, Staahl BT, Chen L, Kuo A et al. An embryonic stem cell chromatin remodeling complex, esBAF, is essential for embryonic stem cell self-renewal and pluripotency. Proc Natl Acad Sci USA 2009; 106: $5181-5186$.

18 Lessard J, Wu JI, Ranish JA, Wan M, Winslow MM, Staahl BT et al. An essential switch in subunit composition of a chromatin remodeling complex during neural development. Neuron 2007; 55: 201-215.

19 Yoo AS, Staahl BT, Chen L, Crabtree GR. MicroRNA-mediated switching of chromatin-remodelling complexes in neural development. Nature 2009; 460: 642-646.

20 Ambasudhan R, Talantova M, Coleman R, Yuan X, Zhu S, Lipton SA et al. Direct reprogramming of adult human fibroblasts to functional neurons under defined conditions. Cell Stem Cell 2011; 9: 113-118.

21 Yoo AS, Sun AX, Li L, Shcheglovitov A, Portmann T, Li Y et al. MicroRNAmediated conversion of human fibroblasts to neurons. Nature 2011; 476 228-231.

22 Tea JS, Luo L. The chromatin remodeling factor Bap55 functions through the TIP60 complex to regulate olfactory projection neuron dendrite targeting. Neural Dev 2011; 6: 5. 
23 Park J, Wood MA, Cole MD. BAF53 forms distinct nuclear complexes and functions as a critical c-Myc-interacting nuclear cofactor for oncogenic transformation. Mol Cell Biol 2002; 22: 1307-1316.

24 Loe-Mie Y, Lepagnol-Bestel A-M, Maussion G, Doron-Faigenboim A, Imbeaud S, Delacroix $\mathrm{H}$ et al. SMARCA2 and other genome-wide supported schizophrenia-associated genes: regulation by REST/NRSF, network organization and primate-specific evolution. Hum Mol Genet 2010; 19: 2841-2857.

25 Lüscher C, Nicoll RA, Malenka RC, Muller D. Synaptic plasticity and dynamic modulation of the postsynaptic membrane. Nat Neurosci 2000; 3: 545-550.

26 Matsuzaki M, Honkura N, Ellis-Davies GCR, Kasai H. Structural basis of long-term potentiation in single dendritic spines. Nature 2004; 429: 761-766.

27 Murakoshi $H$, Wang $H$, Yasuda R. Local, persistent activation of Rho GTPases during plasticity of single dendritic spines. Nature 2012; 472: 100-104.

28 Okamoto K-I, Nagai T, Miyawaki A, Hayashi Y. Rapid and persistent modulation of actin dynamics regulates postsynaptic reorganization underlying bidirectional plasticity. Nat Neurosci 2004; 7: 1104-1112.

29 Huang Y-Y, Kandel ER. 5-Hydroxytryptamine induces a protein kinase A/ mitogen-activated protein kinase-mediated and macromolecular synthesisdependent late phase of long-term potentiation in the amygdala. J Neurosci 2007; 27: 3111-3119.

30 Ramachandran B, Frey JU. Interfering with the actin network and its effect on long-term potentiation and synaptic tagging in hippocampal CA1 neurons in slices in vitro. J Neurosci 2009; 29: 12167-12173.

31 Zhou Q, Xiao M, Nicoll RA. Contribution of cytoskeleton to the internalization of AMPA receptors. Proc Natl Acad Sci USA 2001; 98: 1261-1266.

32 Chen LY, Rex CS, Casale MS, Gall CM, Lynch G. Changes in synaptic morphology accompany actin signaling during LTP. J Neurosci 2007; 27 : 5363-5372

33 Fedulov V, Rex CS, Simmons DA, Palmer L, Gall CM, Lynch G. Evidence that long-term potentiation occurs within individual hippocampal synapses during learning. J Neurosci 2007; 27: 8031-8039.

34 Vo N, Klein ME, Varlamova O, Keller DM, Yamamoto T, Goodman RH et al. A cAMP-response element binding protein-induced microRNA regulates neuronal morphogenesis. Proc Natl Acad Sci USA 2005; 102: 16426-16431.

35 Hansen KF, Sakamoto K, Wayman GA, Impey S, Obrietan K. Transgenic miR132 alters neuronal spine density and impairs novel object recognition memory. PLoS One 2010; 5: e15497.

36 Hansen KF, Karelina K, Sakamoto K, Wayman GA, Impey S, Obrietan K. miRNA-132: a dynamic regulator of cognitive capacity. Brain Struct Funct 2013; 218: 817-831.

37 Wang R-Y, Phang R-Z, Hsu P-H, Wang W-H, Huang H-T, Liu IY, In vivo knockdown of hippocampal miR-132 expression impairs memory acquisition of trace fear conditioning. Hippocampus 2013; 23: 625-633.

38 Wayman GA, Davare M, Ando H, Fortin D, Varlamova O, Cheng H-YM et al. An activity-regulated microRNA controls dendritic plasticity by downregulating p250GAP. Proc Natl Acad Sci USA 2008; 105: 9093-9098.
39 Edwards DC, Sanders LC, Bokoch GM, Gill GN. Activation of LIM-kinase by Pak1 couples Rac/Cdc42 GTPase signalling to actin cytoskeletal dynamics. Nat Cell Biol 1999; 1: 253-259.

40 Impey S, Davare M, Lasiek A, Fortin D, Ando H, Varlamova 0 et al. Molecular and cellular neuroscience. Mol Cell Neurosci 2010; 43: 146-156.

41 Schratt GM, Tuebing F, Nigh EA, Kane CG, Sabatini ME, Kiebler M et al. A brain-specific microRNA regulates dendritic spine development. Nature 2006; 439: 283-289.

42 Szerlong $H$, Hinata K, Viswanathan R, Erdjument-Bromage $H$, Tempst $P$, Cairns BR. The HSA domain binds nuclear actin-related proteins to regulate chromatin-remodeling ATPases. Nat Struct Mol Biol 2008; 15: 469-476.

43 Oma Y, Nishimori K, Harata M. The brain-specific actin-related protein ArpN alpha interacts with the transcriptional co-repressor CtBP. Biochem Biophys Res Commun 2003; 301: 521-528.

44 Aizawa H, Hu S-C, Bobb K, Balakrishnan K, Ince G, Gurevich I et al. Dendrite development regulated by CREST, a calcium-regulated transcriptional activator. Science 2004; 303: 197-202.

45 Qiu Z, Ghosh A. A calcium-dependent switch in a CREST-BRG1 complex regulates activity-dependentgene expression. Neuron 2008; 60: 775-787.

46 Deisseroth K, Mermelstein PG, Xia H, Tsien RW. Signaling from synapse to nucleus: the logic behind the mechanisms. Curr Opin Neurobiol 2003; 13: 354-365.

47 Matamales M. Neuronal activity-regulated gene transcription: how are distant synaptic signals conveyed to the nucleus? F1000Res 2012; 1 : 69.

48 Shen X, Xiao H, Ranallo R, Wu W-H, Wu C. Modulation of ATP-dependent chromatin-remodeling complexes by inositol polyphosphates. Science 2003; 299: 112-114.

49 Steger DJ, Haswell ES, Miller AL, Wente SR, O'Shea EK. Regulation of chromatin remodeling by inositol polyphosphates. Science 2003; 299: 114-116.

50 Rando OJ, Zhao K, Janmey P, Crabtree GR. Phosphatidylinositol-dependent actin filament binding by the SWISNF-like BAF chromatin remodeling complex. Proc Natl Acad Sci USA 2002; 99: 2824-2829.

51 Staahl BT, Crabtree GR. Creating a neural specific chromatin landscape by npBAF and nBAF complexes. Curr Opin Neurobiol 2013; 23: 903-913.

(c) (1) $(\Theta)$ This work is licensed under a Creative Commons Attribution-NonCommercial-NoDerivs 3.0 Unported License. The images or other third party material in this article are included in the article's Creative Commons license, unless indicated otherwise in the credit line; if the material is not included under the Creative Commons license, users will need to obtain permission from the license holder to reproduce the material. To view a copy of this license, visit http://creativecommons.org/licenses/by-nc-nd/3.0/ 